\section{Tribune du président de la SSDV Forum des Präsidenten der SGDV}

\author{
Mesdames, Messieurs, chers Collègues
}

Dans ce numéro, je tiens à attirer votre attention sur la correspondance que j'ai échangée avec M. O. Piller, directeur de l'OFAS, concernant les expertises, rémunérations et certificats relevant du dermatologue. A plusieurs reprises, ces questions ont été discutées au sein du Comité et le Dr F. Gilliet de Bellinzone m'a écrit à ce sujet. Le médecin responsable de la SUVA m'a répondu dans le sens de $\mathrm{M}$. O. Piller et a donc confirmé mes propositions. Ce médecin peut être contacté en cas de problèmes.

Je choisis de publier également une lettre concernant le certificat d'aptitude technique pour le traitement au laser car il permet de maintenir le contrôle de qualité dans ce domaine. Je souhaite remercier le Dr H. Perroud pour son engagement.

Enfin, je vous soumets une lettre du Dr C. Sigg, du groupe de travail Andrologie de notre société, et conseille vivement la lecture de sa publication «Synopsis der medikamentösen Therapie der männlichen Infertilität», à tous ceux qui pratiquent l'andrologie ou à ceux qui, de temps en temps, ont à donner ce genre de conseils.

Avec mes salutations les meilleures.

Prof. R.G. Panizzon

\section{Liebe Kolleginnen und Kollegen}

In dieser Nummer möchte ich den Briefwechsel mit Herrn O. Piller, Direktor des GSV, veröffentlichen. Er betrifft die Punkte Gutachten, Entschädigungen und Zeugnisse. Im Vorstand wurde schon mehrmals darüber diskutiert, und es liegt auch eine entsprechende Dokumentation seitens von Dr. F. Gilliet, Bellinzona,vor. Im gleichen Sinne wie Herr O. Piller hat mir auch der ärztliche Direktor der SUVA geantwortet; er kann bei eventuellen Schwierigkeiten kontaktiert werden.

Wir publizieren auch ein Schreiben von Dr. H. Perroud betreffend Fertigkeitsausweis für die Laserbehandlung. Dieser Ausweis ist sehr wichtig und eine Garantie für die Qualitätssicherung in diesem Bereich. An dieser Stelle möchte ich Dr. H. Perroud für sein Engagement bestens danken.

Schliesslich publiziere ich gerne auch den Brief von Dr. C. Sigg von der Arbeitsgruppe Andrologie unserer Gesellschaft. Seine Schrift «Synopsis der medikamentösen Therapie der männlichen Infertilität» kann ich allen mit der Andrologie Beschäftigten empfehlen, aber auch all denjenigen, die solche $\mathrm{Pa}-$ tienten informieren müssen.

Mit besten Grüssen

Prof. R.G. Panizzon

\section{Expertises/rémunérations/certificats}

Lettre à M.O. Piller, Directeur OFAS

\section{Monsieur le Directeur}

Par ces lignes, je me permets de vous écrire concernant les points suivants.

1) Ces derniers mois, j'ai été contacté par plusieurs médecins au sujet de prestations pour des expertises effectuées par des praticiens non dermatologues. Or, il s'agissait de cas qui relevaient de dermatologues. Je pense que cette démarche n'est pas correcte. En effet, si un deuxième avis sur une maladie de la peau est nécessaire et que cette dermatose est traitée par un dermatologue, il n'est pas question qu'un médecin d'une autre spécialité produise une expertise.

2) Pour toutes prestations adressées à une assurance comme la vôtre, je considère comme normal que le médecin soit rémunéré d'après la prestation, soit un certificat, soit une expertise, mais aussi par exemple une documentation photographique, et cette rémunération doit être en relation avec les coûts de cette prestation (p. ex. photographies).

3) Si l'assurance demande un avis élaboré sur un cas, il est clair que la requête va dans le sens d'une expertise (et le travail doit être rétribué comme tel) et non d'un petit rapport (pour épargner des frais).

En vous remerciant d'avance de votre collaboration et dans l'attente de vos nouvelles, je vous prie de croire, Monsieur le Directeur, à l'assurance de mes sentiments les meilleurs.

Prof. R.G. Panizzon
Réponse de M.O. Piller

\section{Monsieur le Professeur}

Votre correspondance citée en exergue a retenu toute mon attention et je vous en remercie.

S'agissant des trois points soulevés dans votre courrier, je vous fais volontiers part de ce qui suit.

1) Vous avez entièrement raison, il n'est pas correct de demander un deuxième avis pour des expertises à des praticiens non dermatologues, lorsqu'il s'agit de cas qui relèvent de la dermatologie.

2) Il est en effet normal que le médecin soit rémunéré d'après la prestation. Toutefois, s'agissant de la documentation photographique, l'assurance ne la rembourse que si c'est elle qui l'a demandée.

3) Un avis élaboré sur un cas requis par l'assurance doit être remboursé en conséquence (dans le sens d'une expertise et non pas d'un petit rapport).

J'espère que ces quelques lignes pourront vous être utiles. Je vous prie de recevoir, Monsieur le Professeur, mes salutations les meilleures.

\section{O. Piller}




\section{Certificat d'aptitude technique pour le traitement au laser}

Cher Président et Ami

Je te remercie d'avoir soulevé la question des assistants en formation.

Le règlement des dispositions transitoires est aussi applicable pour les assistants à condition

qu'ils aient au moins 2 ans d'expérience pratique avec les lasers et

qu'ils aient exécuté au moins 50 interventions.

Pour les autres qui n'ont pas encore cette expérience, le programme du certificat précise les exigences: cours de base théorique (p. ex. Röntgenkurs) et assistance au nombre requis d'interventions au laser.

Nous invitons donc les médecins assistants de prendre connaissance du programme de formation et de demander le certificat dès que possible.

Les cliniques universitaires de dermatologie seront reconnues dès la séance d'août 2001 par la Laserkommission comme place de formation pour les traitements au laser.

En restant à ta disposition pour tout renseignement complémentaire, je t'adresse, cher Ami, mes cordiales salutations.

Dr Henri M. Perroud

\section{Synopsis der medikamentösen Therapie der männlichen Infertilität}

\section{Lieber Renato}

Im Namen der Schweizerischen Gesellschaft für Andrologie möchte ich dir gerne zwei Exemplare der neu herausgekommenen Schrift zur Synopsis der medikamentösen Therapie in der Andrologie überlassen. Vielleicht findet diese medikamentöse Wegleitung das Interesse der in der andrologischen Sprechstunde beschäftigten Kollegen und Kolleginnen.

Die Schweizerische Gesellschaft für Andrologie stellt auch weitere Informationsschriften zur Verfügung: neu eine Patienteninformation zur intrauterinen Insemination sowie Informationen über Aging-Male und Testosteron-Substitution.

Falls deine Mitarbeiter dies wünschen, können wir die Schriften gerne zur Verfügung stellen.

An dieser Stelle möchte ich die andrologisch interessierten Kollegen auf die Webseite www.andrologie.ch/www.männerheilkunde.ch hinweisen.

Mit freundlichen Grüssen

Dr. C. Sigg 